\title{
Importance of breeding mulberry trees under vegetative (in vitro) methods in high-quality silk production in Uzbekistan
}

\author{
Shavkat Umarov ${ }^{1, *}$, Yorkinoy Mirzaeva ${ }^{1}$, Khurshid Yalgashev $^{1}$, Khurshida Fozilova ${ }^{1}$, and \\ Javokhir Khaydaraliev ${ }^{1}$ \\ ${ }^{1}$ Tashkent State Agrarian University, University str., 2, Tashkent province, Uzbekistan, 100140
}

\begin{abstract}
In the first direction of this article, the new Marhamat, Mustaqillik-18 and Jararik-12 varieties of mulberry have economic value characteristics, such as leaf stem size, leaf length, leaf width, leaf stem length, leaf weight, leaf stem weight, leaf stem weight, and net leaf weight. In addition, the systems of mulberry silkworms Liniya-101 and Liniya-205 were cultivated with Marhamat, Jararik-12 and Mustaqillik-18 and Tajik seedless varieties of mulberry. The second direction of the study was the experiments on in vitro propagation of new varieties of tobacco, and the cost-effectiveness of testing in the conditions of production of positive data obtained on the basis of the results of 2017-2020. At the same time, it can be seen that the economic efficiency of mulberry varieties grown and introduced in vitro is $4,695,300 \mathrm{UZS}$, net profit is $1,577,500 \mathrm{UZS}$, and the size of profitability is $39.6 \%$ contrast to the comparator.
\end{abstract}

\section{Introduction}

Today, live silk cocoon raw materials are produced in more than 20 countries around the world, as well as mulberry fruit is grown in more than a hundred countries for woodworking, landscaping, landscape design, medicine and mainly for the large-scale mulberry silkworm food base $[1,2]$. At present, mulberry is cultivated on 626,000 hectares in the People's Republic of China, 280,000 hectares in India, and more than 35,000 hectares in Thailand and Brazil. Today, $85-90 \%$ of mulberry seedlings are propagated vegetatively in the People's Republic of China, 75-80\% in India, 98\% in Japan, and 4-5\% in Uzbekistan $[3,4]$. The world annually produces $800-850$ thousand tons of raw cocoons and produces an average of 177,832,000 tons of silk fiber per year. The People's Republic of China accounts for $82.1 \%$ of the silk production, India for $16.1 \%$, Uzbekistan for $0.6 \%$, Thailand for $0.4 \%$, Brazil for $0.3 \%$, and other silk-producing countries for the remaining $0.5 \%$. In order to achieve high silk cocoon yields, it is necessary to enrich the silk cocoon food base with new high-yielding and nutritious mulberry varieties and to organize intensive mulberry plantations on their basis [1-5]. Therefore, it is important to create new high-yielding varieties and hybrids of mulberry belonging to the genus Morus alba L., as well as to increase the cocoon productivity of silkworm hybrids by creating new mulberries [6].

${ }^{*}$ Corresponding author: $\underline{\text { ushavkat } @ \text { mail.ru }}$ 
In the leading countries of the world, such as the People's Republic of China and India, where the silk industry is developed, large-leaved mulberry shrubs and semi-shrubs adapted to extreme climatic conditions are being created, using the genetic resources of mulberry and advanced molecular genetic selection techniques. In Uzbekistan, the mulberry gene pool is expanding year by year due to varieties imported from different geographical zones. Examples include mulberry varieties imported from Japan, Korea, China, Bulgaria, Romania, the United States, Georgia, the Russian Federation, Azerbaijan, Vietnam, Ukraine, and Moldova [6, 7, 10].

Data on new mulberry varieties suitable for bivoltin breeds widely cultivated in India and their effective use are provided. According to the expert, mulberry varieties created specifically for bivoltin breeds have a significant impact on cocoon quality and its yield [3].

Researchers believe that the advantage of this variety is its high productivity and suitability for various water shortages. The size of the leaf is $25.0-35.0 \mathrm{~cm}$ and its weight is 8.0-12.0 g. The content of crude protein and carbohydrates is 27.5-28.5 percent and 6.5-7.5 percent [4].

According to the Georgian scientist [5], the silkworm cocoons of the new Nina, Tbilisuri-2, Kvatsikhe, Medgari, Figuruli breeds of mulberry silkworm, suitable for Georgian conditions, have a silk content of 26.0-27.0\% and a total fiber length of 1600$2000 \mathrm{~m}$. Most importantly, these breeds are characterized by their suitability for different climatic conditions [8].

In assessing the nutritional value of mulberry leaves, it was recommended to use its maturity and the relationship between the biological and technological characteristics of mulberry silkworm [9].

The varieties Termez 31, Lixi 4, Lixi 5, A-9-72 in the mulberry collection have been noted by breeders to be able to use them to create new varieties due to their high yield, cold and disease resistance. These varieties have noted the high yield from their 1 bush [10].

Varieties and hybrids of mulberry leaves have been analyzed for their dependence on the content of substances in silkworm assimilation and to what extent they affect the productivity of silkworms $[11,12]$. It was found that when the chemical composition of the leaves was studied, the protein content of the hybrid mulberry leaves was $7.45 \%$ and the free nitrogen content was $1.19 \%$, and the total carbohydrates were $8.0 \%$ [8]. In particular, in such studies aimed at increasing the cocoon productivity of mulberry silkworms, scientists emphasize the importance of leaf quality in increasing the productivity and silkworm performance of promising Liniya-27 and Liniya-28 systems [12-14].

The effects of silkworm strains on stress factors have been shown in ovarian and larval life processes. They argue that with the increase in silkworm and technological performance of new breeds, their embryonic and postembryonic viability may decrease. In their experiments, they achieved positive results by analyzing the viability of the second generation of silkworms obtained under adverse conditions of care of the worms Pearl, Beautiful, Silk-1, Silk-2 and Liniya-27, Liniya-28 [7].

Scientists have been working on vegetative propagation for hundreds of years and have proposed many ways to improve it during this period. A new method of vegetative propagation is micro-clonal propagation, in which the whole plant organism develops as a result of exogenous exposure of plants in vitro. They argue that this method is superior to traditional methods in that it has several advantages [13, 14].

In scientific studies aimed at obtaining high-quality seedlings using biotechnology, they have succeeded in propagating high-yielding and productive vine seedlings. A group of scientists from the University of Taif in Saudi Arabia expressed their views on the benefits of in vitro propagation of native mulberries. Studies have shown that when mulberries are propagated in vitro, the varieties can retain the characteristics of high productivity, disease resistance and high productivity of the mother mulberry $[2,8,14]$. 
In India, Bangladesh, Pakistan, and a number of Asian countries, mulberry trees have been shown to be highly effective in mulberry silkworm feeding, soil erosion, crop protection, mulberry fruit in the food industry, and adult mulberry branch in the woodworking industry. They expressed their views and conducted interesting scientific research on the creation of new varieties and hybrids of mulberry $[6,7]$.

Leading researchers from China, India, Japan, and Korea, where silkworm breeding is developed, have expressed the view that in vitro breeding based on genomic and breeding resources has a number of advantages. They have scientifically substantiated the possibility of modern in vitro microclonal propagation of mulberry varieties, firstly, the cultivation of native seedlings free of bacteria and fungi of the same mother material, and secondly, in vitro propagation of mulberry varieties at any time of the year $[8,10]$.

Effective use of vegetative and molecular-genetic (in-vitro) selection methods in the breeding of mulberry varieties and hybrids, which today are considered essential for the production of silkworms in the leading countries of silkworm breeding, suitable for different climatic stress conditions, high nutritional value, leaf yield and composition. On the basis of the creation of new varieties rich in protein, scientific research is being conducted on the organization of effective intensive mulberries that meet the needs of industrial enterprises. The main goal of the research is to create mulberry varieties that are economically viable, suitable for a variety of extreme conditions, the composition of the leaves is suitable for feeding silkworms.

Analysis of scientific studies conducted by leading scientists shows that the increase in size, weight and silkiness of cocoons, the amount, quality and nutritional value of mulberry leaves fed to mulberry silkworms are important environmental factors, humidity, air temperature. When fed with quality mulberry leaves, it is possible to achieve high biological, cocoon productivity and technological performance of silkworms.

\section{Materials and methods}

Our research has been conducted in two directions.

The first direction is - "Evaluation of the effectiveness of mulberry varieties Marhamat and Jararik-12 on the basis of biological and economic characteristics of silkworms". The following research tasks were identified in this direction;

- to determine the important economic (leaf size, leaf weight, leaf yield) and morphological (number of branches, length of branches, total length of the variety, leaf size, leaf weight, leaf stem length and weight) leaves of mulberry Marhamat and Jararik-12 varieties;

- To determine the chemical composition of the leaves of new selection numbers and varieties of mulberry;

- To prove the influence of mulberry silkworm hybrids on the growth dynamics of the mulberry silkworm during the larval period, as well as the duration of the larval period;

- to prove the effect of leaves of new varieties and selection numbers of mulberry on the viability of silkworm hybrids;

- Economic analysis of the leaves of new varieties and selection numbers of mulberry by studying the size of impact of silkworm hybrids on cocoon productivity and technological properties.

Our research was conducted in the fields of "Jararik Unitary Enterprise" of the Silk Research Institute in Uzbekistan. During the experiments, the leaf size and weight of the new varieties of mulberry Marhamat, Mustaqillik-18, Jararik-12 and comparative Tajik seedless were determined twice a year. The first experimental measurements were carried out on May 10-15, the second measurement on August 10-15. 
For this purpose, 4 plants from each mulberry variety were selected and 30 leaf stems were collected in different parts of them, the total length, width, leaf stem length and leaf weight of each were measured. All identified numbers were recorded in the workbook.

After the weight of the leaves and their size were measured, the leaf stem was measured separately on a series of electronic scales. Its net weight was determined by subtracting the leaf stem from the total surface weight of the actual leaf.

Similar measurements were made in August, the leaf size and weight were determined, and spring and summer samples were compared. The morphological characteristics of mulberry varieties were evaluated and their rating results were determined.

Also, the new selection lines of mulberry silkworm Liniya-101 and Liniya-205 were obtained from silkworms in 4 turns out of 200, and these systems were cultivated with leaves of mulberry Marhamat, Jararik-12, Mustaqillik-18 and comparative Tajik seedless varieties. The silkworm systems in the experimental and comparative variant were kept in a special worm under the same air temperature, humidity, nutrient and worm feeding area.

The second section is - "Development of agrotechnology of in vitro propagation and primary seedling cultivation". The following research tasks were identified throughout this section:

- to determine the conditions for obtaining the initial sterile material for in vitro reproduction of tobacco and the moderate content of carbohydrates, macro-and micronutrients that provide the maximum size of regeneration at the stage of microreproduction;

- optimization of the effect of growth regulators and storage standards for the induction of the process of root formation of varieties and hybrids of mulberry in the process of in vitro micropropagation;

- To determine the varietal characteristics of morphogenesis processes in mulberry tree in vitro propagation and to develop regimes of adaptation to in vitro and soil conditions;

- Approbation of in vitro mulberry seedlings in the open field;

- Evaluation of the cost-effectiveness of seedlings grown in vitro.

In order to implement the above tasks, a number of scientific studies were conducted during 2017-2020.

The main purpose of the study in the first direction was to determine the morphological and economic value of new varieties of mulberry, and to evaluate the economic value of new varieties of mulberry Marhamat, Mustaqillik-18, and Jararik-12.

The aim of our research in the second direction was to determine the different concentrations of different sterilizing substances and to study the optimal nutrient content for micropropagation of tobacco, to determine the most favorable conditions for the growth of plant material.

\section{Results and discussion}

Leaf surface and weight characteristics, which are important economic characteristics of mulberry varieties, were fully analyzed. We know that the larger the leaf surface, the higher the nutritional value of the leaf. The extent to which the above indicators affect the growth and development of silkworms is shown in Table 1. 
Table 1. Leaf size and weight of mulberry varieties (2020)

\begin{tabular}{|c|c|c|c|c|c|c|}
\hline \multirow{2}{*}{ 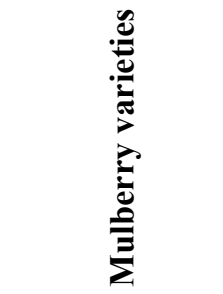 } & \multicolumn{3}{|c|}{ Size of leaves, cm } & \multicolumn{3}{|c|}{ Leaf weight, $g$} \\
\hline & 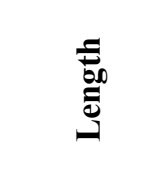 & $\underset{⿱ 乛}{\stackrel{5}{*}}$ & $\underset{\mathscr{D}}{\mathbb{D}}$ & 吾 & $\underset{\mathbb{D}}{\mathbb{D}}$ & 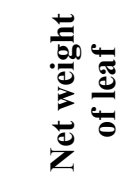 \\
\hline Marhamat & $23.6 \pm 2.09$ & $18.3 \pm 0.35$ & $6.5 \pm 0.06$ & $8.5 \pm 0.61$ & $1.4 \pm 0.22$ & $7.8 \pm 0.29$ \\
\hline Mustakillik-18 & $20.5 \pm 1.03$ & $17.5 \pm 0.32$ & $5.6 \pm 0.17$ & $6.8 \pm 0.43$ & $1.0 \pm 0.32$ & $6.5 \pm 0.23$ \\
\hline Jararik-12 & $19.6 \pm 0.16$ & $16.2 \pm 0.37$ & $5.2 \pm 0.10$ & $6.5 \pm 0.37$ & $0.8 \pm 0.17$ & $5.8 \pm 0.35$ \\
\hline $\begin{array}{l}\text { Tajik seedless } \\
\text { (comparative) }\end{array}$ & $16.5 \pm 0.18$ & $14.3 \pm 0.35$ & $5.1 \pm 0.06$ & $4.1 \pm 0.46$ & $0.6 \pm 0.12$ & $3.5 \pm 0.17$ \\
\hline
\end{tabular}

According to the data in Table 1 above and Figure 1 below, the highest values were observed in the Marhamat variety in terms of leaf size and weight, i.e., while the leaf length and width were $23.6 \times 18.3 \mathrm{~cm}$, its net weight was $7.8 \mathrm{~g}$. Leaf width of Mustaqillik-18 variety was $20.5 \times 17.5 \mathrm{~cm}$, net weight was $6.5 \mathrm{~g}$. We can see that the leaf length of the Jararik-12 variety is $19.6 \times 16.2 \mathrm{~cm}$, while the net weight is $5.8 \mathrm{~g}$. In the comparative Tajik seedless variety, these results averaged $16.5 \times 14.3 \mathrm{~cm}$ and a net weight of $3.5 \mathrm{~g}$. The varieties have a leaf stem of 5.1-6.5 cm in length and weigh from $4.1 \mathrm{~g}$ to $8.5 \mathrm{~g}$ with a leaf stem. The weight of the leaf stem is $1.4 \mathrm{~g}$ in the Marhamat variety, $1.0 \mathrm{~g}$ in the Mustaqillik18 variety, and $0.8 \mathrm{~g}$ in the Jararik-12 variety. In the comparative Tajik seedless variety, this figure was $0.6 \mathrm{~g}$.

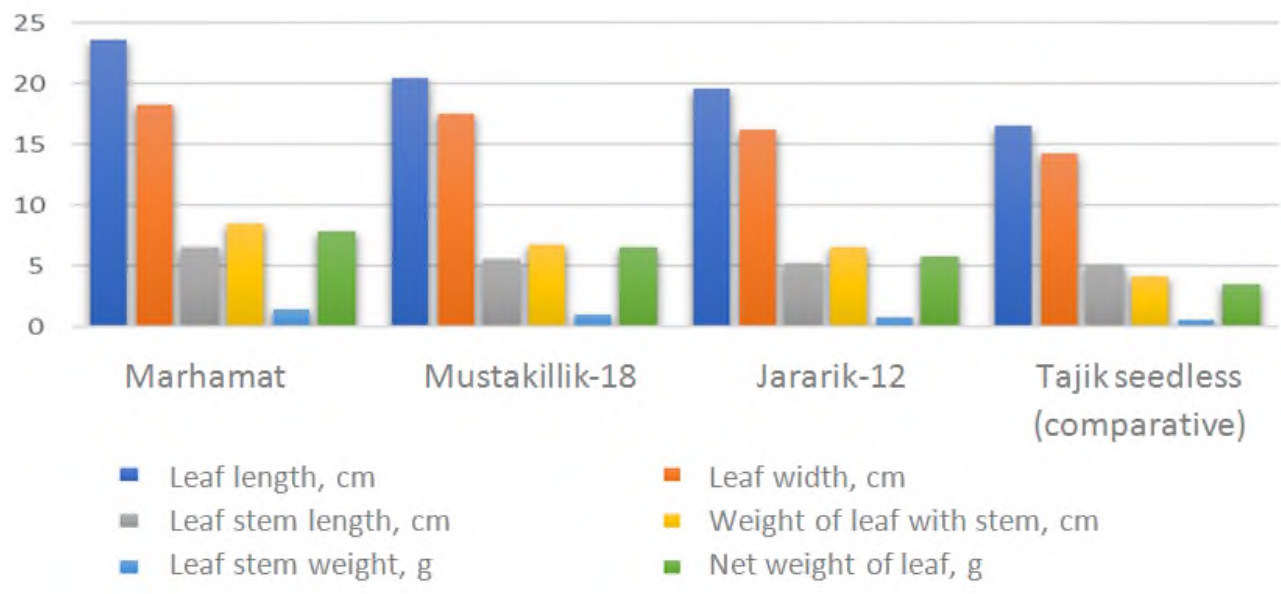

Fig. 1. Leaf size and weight of mulberry varieties

The following table (Table 2) shows the results of our experiments on animated silkworms to test systems with high biological performance, based on the results of care with Liniya-101 and Liniya-205 systems, new Marhamat, Jararik-12 and Mustaqillik-18 and comparative Tajik seedless varieties. 
Table 2. Biological indicators of Liniya-101 and Liniya-205 systems (2020)

\begin{tabular}{|c|c|c|c|c|}
\hline System names & $\begin{array}{c}\text { Larvae } \\
\text { survivability, \% } \\
\bar{X}_{ \pm \mathrm{S}} \bar{x}, \%\end{array}$ & $\begin{array}{c}\text { Incidence, \% } \\
\bar{X}_{ \pm \mathbf{S}} \bar{x}, \%\end{array}$ & $\begin{array}{c}\begin{array}{c}\text { Percentage of } \\
\text { varietal silk } \\
\text { cocoons, }\end{array} \\
\bar{X}_{ \pm \mathrm{S}} \bar{x}, \% \\
\end{array}$ & $\begin{array}{c}\text { Silk } \\
\text { production, \% } \\
\bar{X}_{ \pm \mathrm{S}} \overline{\bar{X}}, \%\end{array}$ \\
\hline \multicolumn{5}{|c|}{ An experimental variant of silkworm mulberry cultivated in the Marhamat variety } \\
\hline Liniya-101 & $90.1 \pm 0.39$ & $2.2 \pm 0.24$ & $92.2 \pm 3.62$ & $22.5 \pm 0.12$ \\
\hline Liniya-205 & $90.4 \pm 0.36$ & $2.8 \pm 0.48$ & $91.6 \pm 3.21$ & $22.5 \pm 0.26$ \\
\hline \multicolumn{5}{|c|}{ An experimental variant of silkworm mulberry cultivated in the Jararik-12 variety } \\
\hline Liniya-101 & $85.8 \pm 0.17$ & $3.4 \pm 0.37$ & $90.7 \pm 3.41$ & $22.0 \pm 0.15$ \\
\hline Liniya-205 & $86.4 \pm 0.35$ & $4.3 \pm 0.32$ & $91.3 \pm 3.11$ & $21.7 \pm 0.04$ \\
\hline \multicolumn{5}{|c|}{ An experimental variant of silkworm mulberry cultivated in the Mustakillik-18 variety } \\
\hline Liniya-101 & $88.1 \pm 0.43$ & $3.9 \pm 0.25$ & $89.1 \pm 3.34$ & $21.4 \pm 0.21$ \\
\hline Liniya-205 & $86.2 \pm 0.67$ & $2.4 \pm 0.50$ & $90.8 \pm 3.14$ & $22.0 \pm 0.08$ \\
\hline \multicolumn{5}{|c|}{ An experimental variant of silkworm mulberry cultivated in the Tajik seedless variety } \\
\hline Liniya-101 & $75.6 \pm 0.12$ & $3.4 \pm 0.23$ & $78.1 \pm 3.01$ & $23.4 \pm 0.30$ \\
\hline Liniya-205 & $75.1 \pm 0.18$ & $4.1 \pm 0.47$ & $76.5 \pm 3.26$ & $23.3 \pm 0.23$ \\
\hline
\end{tabular}

Analysis of the data in Table 2 shows that the worm viability of the silkworm Liniya101 system is $90.1 \%$ when cultivated with the Marhamat variety of mulberry, $85.8 \%$ when cultivated with the Jararik-12 variety of mulberry, and Mustaqillik-18 variety, and 88.1 percent when cared for. The viability of the worms of the Liniya-101 system was $75.6 \%$ when fed with leaves of the comparative Tajik seedless variety. The incidence rate of silkworm larvae of the same variety with the leaves of the Linia-101 selection system is $2.2 \%$, the incidence rate of the larvae of the Linia-205 system is $2.8 \%$, and the percentage of larvae of the Jararik-12 and Mustaqillik-18 varieties is 1, while the incidence of worms ranged from 3.4 to 3.9 percent, while in the Liniya-205 system, the rate was 2.4-4.3 percent. It can be observed that the incidence of silkworms fed on the leaves of our comparative variety was $3.4 \%$ in the worms of the Liniya- 101 system and $4.1 \%$ in the worms of the Liniya-205 system. The share of varietal cocoons was $92.2-92.6 \%$ in the Liniya-101 and Liniya-205 systems maintained by Marhamat, 89.1-90.8\% in the same systems fed with Mustaqillik-18 leaves, and Jararik-12, and in silkworm systems fed with leaves was $90.7-91.3 \%$. The share of varietal cocoons in the systems fed by the leaves of the Tajik seedless variety was $76.5-78.1 \%$. Silkworm rates are $23.3-23.4 \%$ in the lines of Liniya-101 and Liniya-205 with the leaves of the Tajik seedless variety, $22.5 \%$ in the same systems with the leaves of the Blessed variety, Liniya-101 and Lary-12 with the leaves of the Jararik-12 variety. While the silkworm cocoon of the Mustaqillik-18 variety was $21.4-$ $22.0 \%$ in the Liniya-205 system, the silkworm cocoon in the Liniya-101 system was $21.4 \%$, while in the Liniya-205 system it was equal to $22.0 \%$. Due to the low weight of the cocoon in the silkworm systems cared for with the Tajik seedless variety, the silkworm rate was slightly higher than our experimental options.

In our first year experiments, it was observed that the biological performance of silkworm systems fed by Marhamat, Mustaqillik-18 and Jararik-12 varieties of mulberry was higher than the biological performance of worms fed by the Tajik seedless variety.

To study the laws of the moderate composition of growing regulators for micropropagation of mulberry trees in vitro, as well as the moderate ratio of growing regulators for induction of the process of root formation of mulberry varieties and hybrids, to determine the possibility of long-term storage in vitro.

The isolated tertiary meristem cells were predominant based on clonal micropropagation while preserving the genetic characteristics and identity of the offspring. The nutrient medium for the Jararik-9 variety of mulberry selected for the experiments and 
the Uzbek hybrid was prepared with the addition of all components and vitamins of Murasige Skuga (MS). After sterilization, the branches are first transferred to a nutrient medium without phytohormones. When sterilizing agents were used in different amounts, a $0.01 \%$ solution of silver nitrate and $5 \%$ sodium hypochlorite gave good and soft sterilizing results. We were also able to determine the nutrient medium and conditions that allow the sterilized plant parts to grow in vitro. At the same time it was possible to select the optimal conditions for rooting of selected mulberry varieties and hybrids, the emergence of joints, the development of the leaf blade. Growing conditions in the experiments: photo cycle $16 / 8$ hours night - day, illumination 4000-6000 lux, room temperature $24-26^{\circ} \mathrm{C}$, relative humidity $60-70 \%$. In our study, the Uzbek hybrid and the Jararik- 9 variety were better adapted to the artificial nutrient medium than the Balkhi mulberry and 'Shotut' mulberry variety. In our research on the selection of nutrient media for artificial propagation, determination of phytohormone concentrations and cultivation of seedlings in vitro, the first results were obtained by in vitro micropropagation of selected varieties Jararik-9 and hybrid mulberries of Uzbekistan (Figure 2).
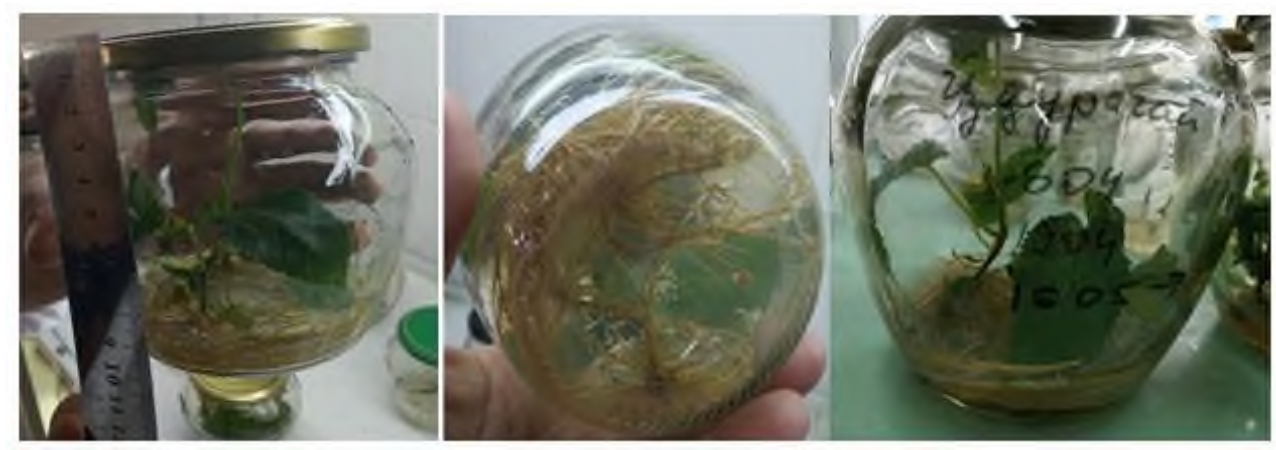

Fig. 2. Micro-propagated seedlings of Jararik-9 variety and hybrid mulberries of Uzbekistan by in vitro method

The first seedlings were prepared in vitro, 4,479 mulberry seedlings were grown in vitro at the Kashkadarya Scientific Experimental Station of the Academician M. Mirzaev Research Institute of Horticulture, Viticulture and Enology in Kashkadarya province, the size of profitability increased by $39.6 \%$ compared to the comparator (Table 3 ). 
Table 3. Indicators of economic efficiency of in vitro cultivation of mulberry seedlings (in Kashkadarya province)

\begin{tabular}{|c|c|c|c|c|c|}
\hline$\#$ & Indicators & Unit & Comparative & $\begin{array}{c}\text { Experiment (in } \\
\text { vitro) }\end{array}$ & $\begin{array}{c}\text { Difference, } \\
+ \text { more; - less }\end{array}$ \\
\hline 1 & $\begin{array}{c}\text { Area sown with mulberry } \\
\text { seeds }\end{array}$ & ha & 0.05 & 0.05 & \\
\hline 2 & $\begin{array}{c}\text { Number of mulberry } \\
\text { seedlings and saplings } \\
\text { grown }\end{array}$ & pcs & 13,500 & 4,479 & $-9,021$ \\
\hline & Cost & UZS & $\mathbf{4 , 5 5 6 , 2 5 0 . 0}$ & $\mathbf{6 , 7 1 8 , 5 0 0 . 0}$ & $\mathbf{2 , 1 6 2 , 2 5 0 . 0}$ \\
\hline 2.1 & $\begin{array}{c}\text { Including the number of } \\
\text { mulberry seedlings }\end{array}$ & pcs & 10,125 & & \\
\hline & Cost & UZS & 250 & & 1,104 \\
\hline & Value & UZS & $2,531,250.0$ & & 900 \\
\hline 2.2 & $\begin{array}{c}\text { Number of mulberry } \\
\text { saplings }\end{array}$ & pcs & 3,375 & 4,479 & $4,693,500$ \\
\hline & Cost & UZS & 600 & 1,500 & \\
\hline & Value & UZS & $2,025,000.0$ & $6,718,500$ & \\
\hline 3 & Costs of growing mulberry seedlings and saplings on the technological map: \\
\hline 3.1 & For 1 ha & UZS & $48,167,350.0$ & $58,200,350.0$ & $10,033,000.0$ \\
\hline 3.2 & Total & UZS & $\mathbf{2 , 4 0 8 , 3 6 7 . 5}$ & $\mathbf{2 , 9 1 0 , 0 1 7 . 5}$ & $\mathbf{5 0 1 , 6 5 0 . 0}$ \\
\hline 4 & $\begin{array}{c}\text { Income at the expense of } \\
1 \text { soum }\end{array}$ & UZS & 1.9 & \multicolumn{3}{|c|}{2.3} & 0.4 \\
\hline 5 & Profit & UZS & $2,147,882.5$ & $3,808,482.5$ & $1,660,600.0$ \\
\hline 6 & Tax (5,0\%) & UZS & $107,394.1$ & $190,424.1$ & $83,030.0$ \\
\hline 7 & Net profit & UZS & $2,040,488.4$ & $3,618,058.4$ & $1,577,570.0$ \\
\hline 8 & Profitability & $\mathbf{9}$ & $\mathbf{8 4 . 7}$ & $\mathbf{1 2 4 . 3}$ & $\mathbf{3 9 . 6}$ \\
\hline
\end{tabular}

\section{Conclusion}

In conclusion, Marhamat and Mustaqillik-18 varieties have the highest rates of spring and summer leaf size and weight, it is necessary to further improve these mulberry varieties and recommend them for production. Taking into account the fact that Jararik-12 mulberry variety gave average results on summer leaf size and weight, it is necessary to continue research work on this mulberry variety.

The role of mulberry varieties and hybrids with high economic characteristics in the preservation of the genetic potential of high-yielding silk and cocoon breeds and their emergence in the conditions of their production is incomparable.

At present, all over the world, native seedlings are created from plants propagated in vitro. Despite the high cost of seedlings, the high quality of the mother seedlings fully justifies itself economically in their use. Mother seedlings are twice as likely to be obtained from in vitro plants, as well as their quality, disease resistance, and short yields and high yields compared to traditional propagated seedlings. 


\section{References}

1. H. Taha, U. M. Ghazy, A. M. M. Gabr, A. A. A. EL-Kazzaz, E. A. M. M. Ahmed, K. M. Haggag, Bulletin of the National Research Centre 44, 1-9 (2020)

2. T. Sarkar, T. Mogili, S. G. Doss, V. Sivaprasad, Biotechnological approaches for medicinal and aromatic plants, 467-487 (2018)

3. P. Jaya, G. Srinivasa, In: International scientific-technical conference, 67-72 (2017)

4. C. Tang, G. Luo, Z. Wang, F. Dai, CLISERI, 19 (2017)

5. N. Kandelaki, CLISERI, 57 (2017)

6. N. Rajabov, SH. Umarov, B. Nasirillaev, KH. Fozilova, Solid State Technology 63(4), 150-159 (2020)

7. SH. Umarov, B. Nasirillaev, N. Rajabov, M. Jumaniyozov, A. Batirova, S. Khuzhamatov, International journal of scientific \& technology research 9(3), 863-866 (2020)

8. K. H. Dhanyalakshmi, H. V. Chaithra, R. S. Sajeevan, K. N. Nataraja, Genetically Modified Crops, 221-236 (2020)

9. T. Sarkar, T. Mogili, V. Sivaprasad, 3 Biotech 7(3), 1-14 (2017)

10. X. Wan, M. Lei, T. Chen, Y. Tan, J. Yang, Science of the Total Environment 599, 1867-1873 (2017)

11. A. M. Giacomin, J. B. Garcia Jr, W. F. Zonatti, M. C. Silva-Santos, M. C. Laktim, J. Baruque-Ramos, Procedia engineering 200, 89-95 (2017)

12. B. Nasirillaev, SH. Umarov, Bulletin of Agrarian Sciences (Uzbekistan) 4(74), 95-98 (2018)

13. A. M. Giacomin, J. B. Garcia Jr, W. F. Zonatti, M. C. Silva-Santos, M. C. Laktim, J. Baruque-Ramos, IOP Publications 254(19), 192008 (2017)

14. M. Akram, F. Aftab, Pak. J. Bot 44, 285-289 (2012) 